University of Nebraska - Lincoln

DigitalCommons@University of Nebraska - Lincoln

Faculty Publications from the Department of Electrical \& Computer Engineering, Department Electrical and Computer Engineering

6-1-1993

\title{
Spectroscopic ellipsometry studies of HF treated Si (100) surfaces
}

Huade Yao

University of Nebraska-Lincoln

John A. Woollam

University of Nebraska-Lincoln, jwoollam1@unl.edu

Samual A. Alterovitz

NASA Lewis Research Center, Cleveland, Ohio

Follow this and additional works at: https://digitalcommons.unl.edu/electricalengineeringfacpub

Part of the Electrical and Computer Engineering Commons

Yao, Huade; Woollam, John A.; and Alterovitz, Samual A., "Spectroscopic ellipsometry studies of HF treated Si (100) surfaces" (1993). Faculty Publications from the Department of Electrical and Computer Engineering. 45.

https://digitalcommons.unl.edu/electricalengineeringfacpub/45

This Article is brought to you for free and open access by the Electrical \& Computer Engineering, Department of at DigitalCommons@University of Nebraska - Lincoln. It has been accepted for inclusion in Faculty Publications from the Department of Electrical and Computer Engineering by an authorized administrator of DigitalCommons@University of Nebraska - Lincoln. 


\title{
Spectroscopic ellipsometry studies of HF treated Si (100) surfaces
}

\author{
Huade Yao and John A. Woollam \\ University of Nebraska, Center for Microelectronic and Optical Materials Research, \\ and Department of Electrical Engineering, Lincoin, Nebraska 68588-0511
}

Samuel A. Alterovitz

NASA Lewis Research Center, Cleveland, Ohto 44135

(Received 26 October 1992; accepted for publication 3 April 1993)

\begin{abstract}
Both ex situ and in situ spectroscopic ellipsometry (SE) measurements have been employed to investigate the effects of HF cleaning on Si surfaces. The hydrogen-terminated (H-terminated) $\mathrm{Si}$ surface was modeled as an equivalent dielectric layer, and monitored in real time by SE measurements. The SE analyses indicate that after a 20-s 9:1 HF dip without rinse, the $\mathrm{Si}(100)$ surface was passivated by the hydrogen termination and remained chemically stable. Roughness of the HF-etched bare Si (100) surface was observed, in an ultrahigh vacuum (UHV) chamber, and analyzed by the in situ SE. Evidence for desorption of the H-terminated Si surface-layer, after being heated to $\sim 550^{\circ} \mathrm{C}$ in the UHV chamber, is presented and discussed. This is the first use of an ex situ and in situ real-time, nondestructive technique capable of showing state of passivation, the rate of reoxidation, and the surface roughness of the H-terminated Si surfaces.
\end{abstract}

In the fabrication of ultralarge-scale integrated circuits, preparation of native oxide free Si surfaces, the monitor and control of Si surface passivation and reoxidation are extremely important issues. Aqueous $\mathrm{HF}$ etching of $\mathrm{Si}$ surfaces removes the surface oxide and terminates the $\mathrm{Si}$ surface with atomic hydrogen. ${ }^{1,2}$ The hydrogen termination retards the Si surface oxidation, and protects the surface from chemical attack. ${ }^{3,4}$ Therefore, the HF cleaning of Si surfaces has received increasing attention. However, the properties of hydrogen-terminated (H-terminated) Si surfaces under various conditions, and the degree of surface passivation and reoxidation are still under investigation..$^{4-7}$ In this letter, we report results of ex situ and in situ spectroscopic ellipsometry (SE) studies of HF cleaned $\mathrm{Si}$ (100) surfaces.

SE is a surface-sensitive, nondestructive optical technique used to characterize surface overlayer thicknesses, multilayer structures, optical constants of bulk materials, and surface changes. ${ }^{8,9}$ Ellipsometry determines the complex ratio of reflectance $R_{p}$ to $R_{s}$, defined as

$$
\rho=R_{p} / R_{s}=\tan (\psi) e^{i \Delta},
$$

where $R_{p}$ and $R_{s}$ are the reflection coefficient of light polarized parallel to $(p)$ or perpendicular to $(s)$ the plane of incidence, and the values of $\tan (\psi)$ and $\Delta$ are the amplitude and phase of the complex ratio.

The pseudodielectric function $\langle\epsilon\rangle$ can be obtained from the ellipsometrically measured values of $\rho$, assuming a two-phase model (ambient/substrate) ${ }^{8}$ regardless of the possible presence of surface overlayers. For samples with surface overlayers or multilayer structures, SE data must be numerically fitted according to an assumed model (e.g., a three-phase model: ambient/surface overlayer/ substrate). Assuming such a model, values of $\psi^{c}\left(h v_{i}, \Phi_{j}\right)$ and $\Delta^{c}\left(h v_{i}, \Phi_{j}\right)$, defined as in Eq. (1), are calculated. Here $h v$ is the photon energy and $\Phi$ is the external angle of incidence. A regression analysis is established to vary the model parameters (e.g., layers thicknesses) until the calculated and experimental values match as closely as possi- ble. This process is done by minimizing the mean square error (MSE) function as described in Refs. 10 and 11. In our study, ex situ and in situ SE measurements were made using a Woollam Co. Variable Angle Spectroscopic Ellipsometer (VASE ${ }^{\mathrm{TM}}$ ), which was equipped with a beamchopped, rotating analyzer to increase the stray light rejection and signal to noise ratio.

Si (100) surfaces covered with native oxide from a virgin $p$-type wafer of $14-22 \Omega \mathrm{cm}$ resistivity were employed to study the effects of HF treatments on Si. A piece from the wafer was dipped in 9:1 (volume ratio of deionized water to $49 \% \mathrm{HF}$ ) HF solution for approximately $20 \mathrm{~s}$ with no rinse. SE measurements were made in air, at a $75^{\circ}$ angle of incidence, before and after the HF dip. During the measurement, the automated polarizer azimuth angle $P$ was set to vary with changes of the measured $\psi\left(h v_{i}, \Phi_{j}\right)$ to minimize experimental errors in the ellip-

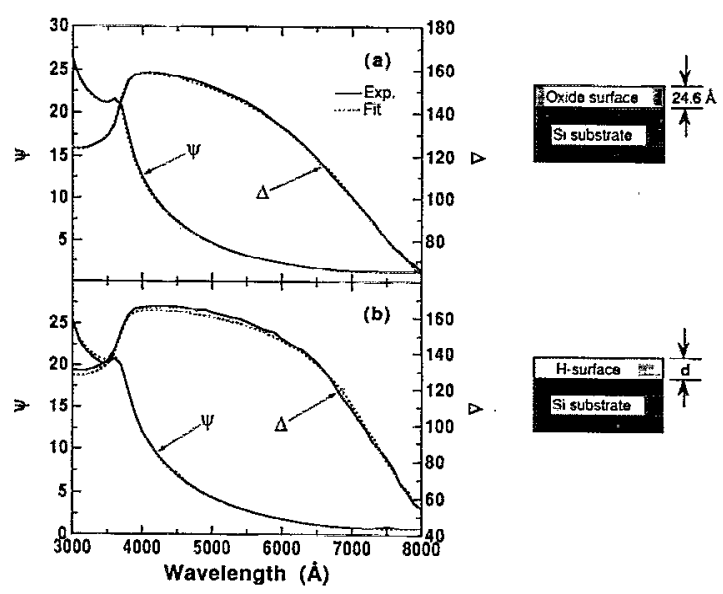

FIG. 1. $\Psi$ and $\Delta$ values of ex situ SE measurements on a Si (100) surface: (a) before and (b) after the HF treatment. The solid line represents the experimental data, and the dashed line is the best fit of the SE analysis. Assumed models for the SE analysis, in each case, are sketched with the plots. 


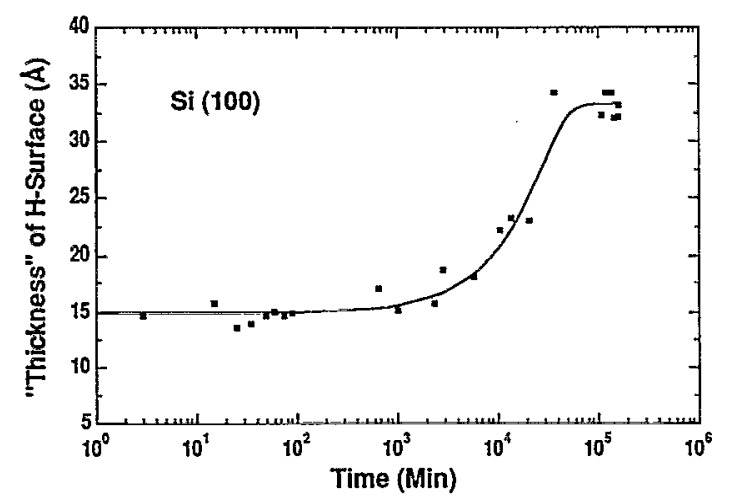

FIG. 2. Changes in effective thickness of the $\mathrm{H}$-terminated Si surface ( $\mathrm{H}$ surface) as a function of time, after the 20-s 9:1 HF dip, monitored by the ex situ SE measurements.

someter system. ${ }^{12}$ It was set such that $P=\psi$ with $P_{\min }=10^{\circ}$. The spectral scan, ranging from 3000 to $8000 \AA$, with an increment of $100 \AA$, was completed within $2-3 \mathrm{~min}$. The $\mathrm{SE}$ analysis indicated that an approximately $25 \AA$ thick native oxide covered the virgin Si surface as shown in Fig. 1(a). After the HF cleaning, the Si native oxides were removed and the Si surface was terminated with $\mathrm{H}$-silicon bonds. ${ }^{1,2}$ This H-terminated Si surface was modeled as an equivalent dielectric layer described by the optical constants of $\mathrm{SiO}_{2}{ }^{13}$ [for the SE analysis shown in Fig. 1(b)]. The optical constants of $\mathrm{Si}$ used for the calculations in the SE regression analysis are from Ref. 14. A typical "thickness" of the H-terminated Si surface (H surface) immediately after the HF cleaning, as indicated by the SE regression analysis, was in the range 14-17 $\AA$. Notice that the thickness referred to here as an $\mathrm{H}$ surface was not the actual thickness of the $\mathrm{H}$-surface layer, but the effective thickness of the modeled equivalent dielectric layer of $\mathrm{SiO}_{2}$, which includes possible $\mathrm{Si}$ surface microroughness after HF etching (as discussed below). The value of this thickness as measured by SE was used to monitor the changes in the $\mathrm{H}$ surface.

SE measurements were made on this $\mathrm{H}$ surface in air at room temperature (RT) over a period of several months after the HF cleaning. Changes in thickness of the $\mathrm{H}$ surface were monitored as a function of time as shown in Fig. 2. The figure shows that the H-terminated Si surface remained unchanged for over $2 \mathrm{~h}$, and very little reoxidation took place within 3-4 days. After two months the reoxidized Si surface layer saturates at a thickness of $\sim 33 \AA$, which is thicker than the native oxide before the HF etching. The SE study indicates that remarkable surface passivation has been achieved by the hydrogen termination of $\mathrm{Si}$ surface dangling bonds, which contributes to the retardation of the Si surface oxidation during air exposure. The apparent larger thickness of the reoxidized layer provides a clue to the Si surface roughness after the HF etching.

In situ SE was employed to study changes of the $\mathrm{H}$-terminated $\mathrm{Si}$ surface at elevated temperatures and the bare Si surface conditions after HF etching. During the in situ measurements, the ellipsometer was attached to an UHV chamber, fitted with a pair of low-strain fused-quartz

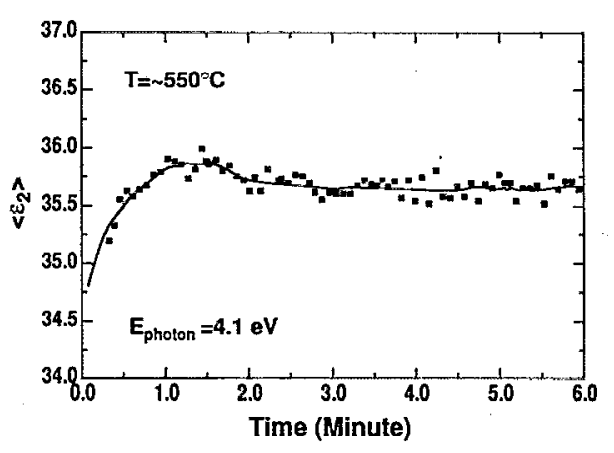

FIG. 3. Real time $\left\langle\epsilon_{2}\right\rangle$ data from the HF-treated $\mathrm{Si}(100)$ surface, inside the UHV chamber, at $\sim 550^{\circ} \mathrm{C}$, near the $E_{2}$ critical-point energy.

windows. ${ }^{11,15} \mathrm{~A} \mathrm{Si}$ (100) sample was introduced into the UHV chamber, immediately after a 20-s 9:1 HF dip, and clamped on a resistor-heater plate that could be rotated and tilted by a rotary drive, inside the chamber. Temperatures were measured and controlled by two $K$-type thermocouples, which were calibrated by an infrared (IR) optical pyrometer. The typical base pressure of the UHV was $\sim 1 \times 10^{-9}$ Torr.

The HF-cleaned $\mathrm{Si}$ (100) surface was heated to $\sim 550^{\circ} \mathrm{C}$ inside the UHV chamber, and real time ellipsometric measurements of one set of $\Psi$ and $\Delta$ data were made periodically in time (about once every $5 \mathrm{~s}$ ) at a photon energy of $\sim 4.1 \mathrm{eV}$, corresponding to the critical point energy $E_{2}$ (i.e., $\sim 4.1 \mathrm{eV}$ for $\mathrm{Si}$ at $\sim 550^{\circ} \mathrm{C}$ ), ${ }^{16}$ while maintaining the sample at $\sim 550^{\circ} \mathrm{C}$. These data were converted simultaneously to a pseudodielectric function $\langle\epsilon\rangle=\left\langle\epsilon_{1}\right\rangle$ $+i\left\langle\epsilon_{2}\right\rangle$. In general, it has been established ${ }^{9,11,15}$ that the peak value of the imaginary part $\left\langle\epsilon_{2}\right\rangle$ in semiconductors is very sensitive to the presence of surface overlayers (e.g., $\mathbf{H}$ surface) and any possible surface defects such as surface microscopic roughness. A reduction of the thickness of surface overlayer or surface roughness leads to increasing values of $\left\langle\epsilon_{2}\right\rangle$. The highest value $\left\langle\epsilon_{2}\right\rangle$ corresponds to the cleanest and smoothest bulk surface condition. Therefore, changes in the $\mathbf{H}$ surface induced at elevated temperatures can be monitored in real time by measuring the changes of $\left\langle\epsilon_{2}\right\rangle$.

Figure 3 shows changes in $\left\langle\epsilon_{2}\right\rangle$ in real time at
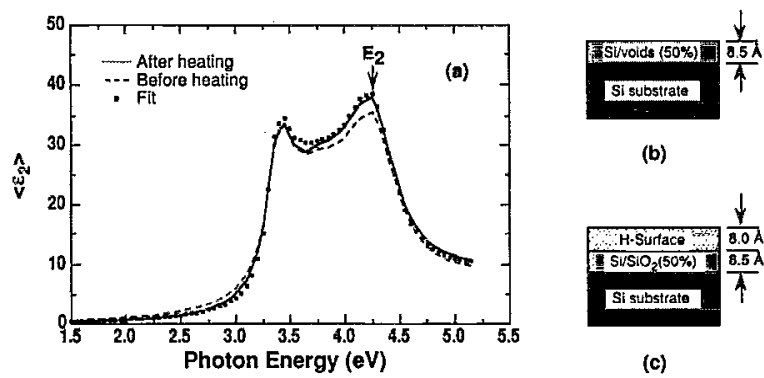

(b)

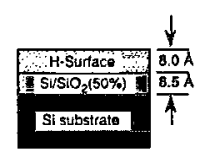

(c)

FIG. 4. (a) $\left\langle\epsilon_{2}\right\rangle$ spectrum of HF-treated Si at RT, measured by in situ $\mathrm{SE}$, before (dashed line) and after (solid line) being heated to $\sim 550^{\circ} \mathrm{C}$ in the UHV chamber. The dotted line is the fit for the rough Si surface model, sketched in (b). (c) A sketch of the H-surface structure, containing the surface roughness formed by HF etching. 
$\sim 550^{\circ} \mathrm{C}$. An obvious increase of $\left\langle\epsilon_{2}\right\rangle$ within the first minute at $\sim 550^{\circ} \mathrm{C}$ indicates the desorption of $\mathrm{H}$-terminated $\mathrm{Si}$ surface at $\sim 550^{\circ} \mathrm{C}$. A flat plateau followed the desorption of the $\mathrm{H}$ surface indicates a stabilized Si surface. The plateau remained unchanged during further extended heating. This also suggests that there is no evidence of the surface quality deterioration (e.g., increasing surface roughness induced by heating) at this elevated temperature. ${ }^{15}$

SE data for the H-terminated Si surfaces were taken at RT before and after the heating, as shown in Fig. 4(a). A comparison of the two $\left\langle\epsilon_{2}\right\rangle$ spectra at RT, shows a $\sim 7 \%$ higher $\left\langle\epsilon_{2}\right\rangle$ peak value at the $E_{2}$ critical point energy $(\sim 4.3 \mathrm{eV}$ at RT) after the heating. This further confirms the desorption of the $\mathrm{H}$ surface after heating to $\sim 550^{\circ} \mathrm{C}$. The evidence for desorption observed in this experiment is consistent with results obtained by thermally stimulated desorption measurements as described in Ref. 5.

The RT $\left\langle\epsilon_{2}\right\rangle$ peak value at the $E_{2}$ energy after the desorption of the $\mathrm{H}$ surface, as shown in Fig. 4(a) (solid line), is considerably lower than the known value $(\sim 46)$ of $\mathrm{Si}$ from literature. ${ }^{14}$ This indicates a roughened Si surface induced by the HF etching. The surface roughness was modeled as a top Si layer containing $50 \%$ voids, as shown in Fig. 4(b). The thickness of this rough Si layer was calculated by the regression analysis, under the assumption of the Bruggeman effective-medium approximation (EMA).${ }^{17}$ Good fit was obtained with a thickness of $8.5 \AA$, as shown in Fig. 4(a) (dotted line). It indicates an approximate 1-2 monolayer surface microroughness formed from the HF etching.

This model of rough Si surface was applied to characterize the $\mathrm{H}$ surface measured at $\mathrm{RT}$ in vacuum, before the heating, as shown in Fig. 4(c). In this case, the voids were replaced by $\mathrm{SiO}_{2}$ to model the $\mathrm{H}$ termination of the rough Si layer, while the thickness of this rough layer was kept the same as in Fig. 4(b). On top of the rough Si surface, a pure $\mathrm{H}$-surface layer was modeled and described by the optical constants of $\mathrm{SiO}_{2}$, as we have discussed previously. The thickness of $\sim 8 \AA$ of this pure H-surface layer was calculated through the regression analysis. Notice that by modeling a rough interface between the top $H$ surface and $\mathrm{Si}$ substrate, the top pure $\mathrm{H}$ surface has an effective thickness of $\sim 8 \AA$, which is consistent with an expected monolayer $\mathrm{H}$ termination of $\mathrm{Si}$ surface.

Same surface-roughness model [as in Fig. 4(c)] was used to reanalyze the ex situ RT SE measurements shown in Fig. 1. The results show a reduced effective thickness of the initial top $\mathrm{H}$ surface, after the HF dip, of $\sim 5.4 \AA$, and a saturated reoxide layer is $\sim 21.7 \AA$. This value is quite consistent with the thickness of a fresh native oxide surface layer. The small difference between the two initial thicknesses of $\mathbf{H}$ surface (i.e., 8.0 and $5.4 \AA$ ) are likely due to different degrees of surface roughness of the two individual $\mathrm{HF}$ treated Si samples.

In summary, HF treated $\mathrm{Si}(100)$ surfaces were investigated by ex situ and in situ SE measurements. The SE analysis indicated that the $\mathrm{Si}(100)$ surface was well passivated via a $\sim 20$-s 9:1 HF dip with no rinse. Real-time SE data showed evidence for desorption of the H-terminated $\mathrm{Si}$ surfaces at $\sim 550^{\circ} \mathrm{C}$ in the UHV chamber. $\mathrm{Si}(100)$ surface roughness induced by HF etching was observed in a UHV chamber, and analyzed by the in situ SE. It was shown that a $\sim 1-2$ monolayer surface roughness was formed after our HF etching. This unique surface characterization, by in situ SE, provides a useful means of studying and monitoring various Si surface conditions after $\mathrm{HF}$ cleaning.

The authors wish to thank Dr. Y. J. Chabal and AT\&T Bell Laboratories for his helpful discussions. This work was partially supported by NASA-Lewis Grant NAG3-154.

${ }^{1}$ E. Yablanovitch, D. L. Allara, C. C. Chang, T. Gmitter, and T. B. Bright, Phys. Rev. Lett. 57, 249 (1986).

${ }^{2}$ M. Grundner and H. Jacob, Appl. Phys. A 39, 73 (1986).

${ }^{3}$ G. S. Higashi, Y. J. Chabal, G. W. Trucks, and K. Raghavachari, Appl. Phys. Lett. 56, 656 (1990).

${ }^{4}$ P. Jakob, P. Dumas, and Y. J. Chabal, Appl. Phys. Lett. 59, 2968 (1991).

${ }^{5}$ N. Hirashita, M. Kinoshita, I. Aikawa, and T. Ajioka, Appl. Phys. Lett. 56,451 (1990).

${ }^{6}$ M. Morita, T. Ohmi, E. Hasegawa, M. Kawakami, and M. Ohwada, J. Appl. Phys. 68, 1272 (1990).

${ }^{7}$ P. Jakob and Y. J. Chabal, J. Chem. Phys. 95, 2897 (1991).

${ }^{8}$ R. M. A. Azzam and N. M. Bashara, Ellipsometry and Polarized Light (North-Holland, Amsterdam, 1977).

${ }^{9}$ D. E. Aspnes, in Handbook of Optical Constants of Solids, edited by E. D. Palik (Academic, New York, 1985), p. 89.

${ }^{10}$ G. H. Bu-Abbud, N. M. Bashara, and J. A. Woollam, Thin Solid Films 138, 27 (1986).

${ }^{11}$ H. Yao, P. G. Snyder, and J. A. Woollam, J. Appl. Phys. 70, 3261 (1991).

${ }^{12}$ D. E. Aspnes, J. Opt. Soc. Am. 64, 639 (1974).

${ }^{13}$ H. R. Philipp, Ref. 9, p. 759.

${ }^{14}$ G. E. Jellison, Jr., Opt. Mater. 1, 41 (1992).

${ }^{15}$ H. Yao and P. G. Snyder, Thin Solid Films 206, 283 (1991).

${ }^{16} \mathrm{H}$. Yao (unpublished data).

${ }^{17}$ D. E. Aspnes and J. B. Theeten, Phys. Rev. B 20, 3292 (1979). 